\title{
Key Tasks of Research on Agricultural IOT Technology and Service Innovation
}

\author{
Jifang Liu ${ }^{1, a}$, Jianhua Zhang ${ }^{2, b}$, Shuqing $\mathrm{Han}^{3, \mathrm{c}}$ and Fantao Kong ${ }^{4, \mathrm{~d}}$ * \\ ${ }^{1,2,3,4}$ Agricultural Information Institute, Chinese Academy of Agricultural Sciences/Key Laboratory of \\ Agri-information Service Technology, Ministry of Agriculture, Beijing 100081,China \\ aliujifang@caas.cn, ㅎzhangjianhua@caas.cn, hanshuqing@caas.cn, kkongfantao@caas.cn
}

\begin{abstract}
Keywords: Agricultural informatization; agricultural IOT; IOT Ranch; research emphasis.
Abstract. The application of the Internet of Things, cloud computing, big data, mobile Internet and other modern information technologies in the transformation and upgrading of the whole industrial chain of agriculture is of great significance to accelerate the development of agricultural information and modernization. Relying on the existing research base of the agricultural IOT technology and service innovation team of Chinese Academy of Agricultural Sciences, in accordance with the principle of "Indomitable Spirit", the paper systematically describes the research and development of key technologies, development of technical equipments, promotion and application of technologies needing urgent implementation for IOT in China recently and focuses on core technologies of "IOT Ranch", "IOT Vegetable Garden" and other demand orientation.
\end{abstract}

\section{Introduction}

About the technology and service innovation of agricultural IOT, in recent period, technology research and equipment development in three aspects of R\&D of key technologies of perception and transmission of IOT, development of agricultural IOT technologies and equipments and their promotion and application will be focused, and the breakthrough of the following three key technologies will be made: environmental monitoring technology of "IOT Ranch", animal life body sensing technology and detection technology for cotton diseases and insect pests; 3 sets of livestock and poultry husbandry environmental monitoring technical equipments will be developed and they will be promoted and applied in 7 farms and planting bases in Beijing, Hebei, Shandong and other provinces so as to further enhance the universality and accuracy of IOT technologies and equipments.

\section{R\&D of key technologies of IOT perception and transmission}

Implementation of basic and key core technology research in view of the perception layer and transmission layer of IOT technology is the prerequisite condition to promote healthy and rapid development of agricultural IOT technology. On the basis of extensive investigation and research, regard the demand and problem as the guide to focus on the research in the following three aspects.

R\&D of key technologies of environmental monitoring of "IOT Ranch"

Firstly, carry out the research and development of 16 interface sensor data acquisition technology. In view of the problems of less information of data acquired and incomplete index collected of 8 interface sensor currently used, it is planned to adopt the embedded development technology and design multilayer electronic circuit and access expansion circuit to achieve data acquisition technology connected by 16 sensors. Secondly, carry out the research and development of a variety of wireless transmission modes. For the incomplete coverage of network signals in rural areas, carry out the research on WIFI, Bluetooth, GPRS, 3G / 4G and other wireless transmission modes and improve the reliability of information transmission of IOT; carry out R\&D of new sensor technology of "Four-in-one"; in view of the single collection problem of current sensor of temperature, humidity, light intensity and atmospheric pressure, through the design of circuit and interface, integrate four kinds of sensors to improve their efficiency and reduce their size and power consumption. Thirdly, carry out the research on IOT energy supply method; for the problem of instability of power supply of 
IOT equipments, build solar panel + battery mode to solve the discontinuity and instability of energy supply of IOT.

\section{Research on Key Technologies of perception of livestock and poultry life body}

Firstly, carry out the research on perception method of activity amount of livestock and poultry; in view of the problem that it is difficult to perceive the activity amount of animals, based on the formed Bayesian network and hidden Markov model, by combing the threshold classification algorithm, from the analysis of acceleration information of $\mathrm{X}, \mathrm{Y}$ and Z-axis direction, build the gait calculation model of livestock and poultry and carry out the research on the movement discriminating method under four conditions of jog, walking, trot and gallop of livestock and poultry to achieve accurate statistics on the individual activity amount. Secondly, carry out the research on information perception technology of life body of livestock and poultry. The research on wearable technology for livestock and poultry using the embedded system and PCB circuit board as the framework, and mainly the temperature, activity amount, respiration and heart rate as the index achieves the uninterrupted collection of physiological information monitoring of individual livestock and poultry and intelligently judges their estrus and physiological health status and other information.

\section{Research on Cotton Disease and Pest Detection Method Based on Image Processing}

Firstly, carry out the research on cotton image information analysis method under natural conditions. According to the influence of many factors of field panting of cotton, such as light, background, weather, shadow and others, on the image acquisition, analyze the color, shape and texture change law of leaves and background area under different light and background conditions and select insensitive information to light and weather. Secondly, carry out the research on the image segmentation method of multi-information fusion. By the test of images of the diseased leaves of cotton in complex backgrounds including different light, backgrounds, weather, shadows and others, explore high robust cotton leaf segmentation method so as to achieve accurate segmentation of cotton leaves in complex backgrounds of different light, weeds, soil, straw, mulch film, shadow and others. Thirdly, carry out the research on the method of separation of healthy leaves from diseased leaves of cotton. From the diseased spots, holes, incompleteness, curling and other characteristics, by combining the image segmentation and classification method, develop the method to judge whether the diseases of segmented leaves exist and achieve the separation of healthy leaves from the diseased leaves of cotton so as to provide technical support for remote detection and diagnosis of cotton diseases and insect pests.

\section{Development of agricultural IOT technology and equipment}

Rely on the research on key technologies, use the existing mature technologies comprehensively, carry out the integrated innovation of the development of IOT technology and equipment, focus on the research and development of related technologies and equipments of "IOT Ranch" and "IOT Vegetable Garden" so as to pave the way for transformation and application of achievements.

\section{Development of Livestock Husbandry Environment Information Monitor}

Firstly, carry out the research on sensor technology for the demand for livestock husbandry environment information collection. Analyze the characteristics of acquisition and data transmission of meteorological sensors (including temperature, humidity, light intensity, rainfall, etc.), gas sensor (CO2, NH3, CH4, H2S etc.), design the connection interface of multi-sensor to the device and acquire livestock husbandry environment information. Secondly, carry out the integrated innovation development of the closed livestock and poultry husbandry environment information monitor. For the problems of high power consumption, low efficiency, big volume and difficult installation of existing equipments in the livestock and poultry house and other indoor conditions, develop the miniaturized monitor; it is planned to use the embedded ARM development technology, integrate the system function, crop redundancy function module, re-design the system circuit, further reduce power consumption, reduce the volume of the monitoring equipment, improve the system stability, while integrate 360-degree panoramic wireless camera to achieve real-time monitoring of animals in the 
house. Thirdly, carry out the integrated innovation development of open-air livestock and poultry husbandry environmental information monitoring station. For the feature of complex power supply conditions of open-air husbandry, integrate solar charging control module and high-performance lithium iron phosphate battery module and build solar power supply system to provide stable power output for the monitoring station and make it work around the clock.

\section{Development of equipment system and remote control for "IOT vegetable garden"}

Firstly, carry out the development of equipment system for "IOT vegetable garden". In view of the problems that the vegetable growth cycle is short, requirement for planting environment is high, the plantation management labor intensity of farmers is large and it is not suitable for them to work in hot and humid environment for a long period of time, carry out the research on the equipment and system of the "IOT vegetable garden" to achieve automatic sensing of environmental information. Secondly, develop the remote control equipments. For different demands of different vegetables for water, develop and build automatic monitoring and early warning methods for vegetable garden soil moisture, system integrating 360-degree panoramic wireless cameras, soil temperature and humidity sensors, controllers, collectors, solenoid valves, sprinkler heads, DTU data transmission and other modules, set up the vegetable garden information monitoring platform based on Web + phone APP mode and build the micro landlord intelligent irrigation equipment so as to achieve real-time monitoring for vegetable growing environmental information and growth status and achieve the purpose of remote view and intelligent irrigation and that of timely discovery of pests and diseases, optimize crop growth environment, reduce production costs and improve work efficiency and product quality.

\section{"IOT Ranch" - R\&D of livestock and poultry husbandry environment sewage monitor}

Firstly, carry out the research on the main monitoring index screening of livestock and poultry husbandry sewage. For the status that there are no special livestock and poultry husbandry environment sewage monitoring equipments, analyze the main pollutant compositions of livestock and poultry husbandry, select the main monitoring indicators of the sewage, develop harmful gas and husbandry wastewater monitoring module, and finally integrate livestock and poultry husbandry environmental sewage monitor Secondly, build exhaust gas monitoring module; for miniaturization and integrated harmful gas monitoring module for measuring ammonia, hydrogen sulfide, methane and carbon dioxide, adopt the modular method to design the hardware, unify the sensor interface, achieve PNP of sensor and improve the stability and universal applicability of the device. Thirdly, Design the husbandry wastewater monitoring module. In view of the characteristics of complex composition, high corrosiveness, high concentration of suspended solids of livestock and poultry wastewater, select protective materials, design waterproof and corrosion resistant sealed enclosure, develop the husbandry wastewater monitoring module integrating $\mathrm{pH}$, oxygen content, water conductivity, salinity, flow rate and sensor, combine the core control module and gateway communication module to achieve online automatic monitoring of indicators of harmful gases, such as ammonia, hydrogen sulfide, methane, carbon dioxide, and husbandry wastewater indicators, such as $\mathrm{pH}$, oxygen content, water conductivity, salinity and flow rate.

\section{Promotion and application of agricultural IOT technology and equipment}

Advanced technology and equipment must be validated and tested by practice. The technical performance of "IOT ranch" and "IOT vegetable garden" and related technology solutions can be further improved and maturation of their technologies is promoted on the basis of pilot demonstration, promotion and application.

\section{Promotion and application of "IOT Ranch" technology and equipment}

Firstly, implement the promotion, application and research of IOT equipments. For the field of livestock husbandry, choose beef cattle husbandry enterprises, promote and apply 5 "Open-air livestock and poultry husbandry environmental information stations" in beef cattle farms in Yangxin County, Shandong Province; for the field of poultry farming, choose hen enterprises, promote and 
apply 4 "Closed livestock and poultry husbandry environmental information monitors" in Pinggu District, Beijing. Secondly, carry out the research on the upgrading of IOT technology. For the problems of livestock husbandry environmental monitoring equipments in the actual pilot application, carry out the research on upgrading of technology so that the stability and practicality of livestock husbandry environmental monitoring and control technology and equipment can be further improved. Thirdly, carry out the maturation and upgrading of IOT technology and equipment. Improve the accuracy and efficiency of technologies, shorten the response time of the related technologies, while further strengthen the signal anti-jamming capability of the device, solve the problem of poor adaptation under harsh natural conditions, and implement the user-friendly design for equipment operator interface so as to provide usability of equipment.

\section{Promotion and application of technology and equipment of "IOT vegetable garden"}

Firstly, carry out the research on the "IOT vegetable garden" achievement transformation model. For the field of vegetable planting, select the "Micro-landlord" vegetable garden, carry out technical tests in Laixi County, Shandong Province, implement the device and system platform application evaluation, select 1-2 application bases with more significant achievements as the demonstration sites of "IOT vegetable garden" for key construction. Secondly, carry out the application research on automatic sensing and control of vegetable growth information. For different growth cycles and planting models of leafy, vining and tree-like vegetables, explore the relation of relative position sensing area between different sensors and the leaves and roots of the crops and the soil around them, analyze the degree of obstruction from different plant heights and leaf areas to the sensor signals; start with the transmitting power, transmission frequency and energy supply of the sensor, improve the efficiency and effect of acquisition, perception, transmission and application of the sensor, build the relation model between perceive information and automatic control and achieve automatic supply and regulation of trace elements, water and light needed by vegetable growth.

\section{Research on IOT technology service solutions}

Firstly, carry out the research on IOT technology service solutions. For the technology needs of different enterprises, for example: livestock and poultry husbandry, field planting, vegetable production and others, study and develop appropriate technical solutions, carry out IOT technology service research and meet the needs of the market. Secondly, carry out the research on IOT technology solutions of three-layer integration. According to different agricultural production modes, different geographical regions, production scales, production conditions, personnel ratio and production equipment, from the collection of sound, image and digit in agricultural production, combine the wireless and wired transmission modes, backstage management system, mobile phone terminal means to design a complete set of overall IOT solutions integrating perception, transmission and application layer of IOT as a whole. Thirdly, carry out the research on professional IOT technical solutions. Combine with the agricultural production practice so as to reduce the costs and investment of agricultural production, improve production efficiency and output targets, give full play to the advantages of IOT technology and equipment and build the intelligent management solution of agricultural production IOT so that the agricultural production automation and management become more intelligent. (Fund project: soft science research project of the Ministry of Agriculture, "Information Supervision and Policy Research of Chinese Seed Industry based on the Big Data Perspective" (No. 201607-2), Beijing Natural Science Found Preliminary Exploration Project, "Beijing Pork Market Monitoring and Early Warning Research Based on Multi-Source Data Fusion “(No. 9153023)).

\section{References}

[1] Kong Fantao, Chen Ping, Wang Ping, etc. Practices and Reflections of Agricultural Informatization Construction In China: Exploration and Inspiration Based on the"Liaoning Model"[J] Research of Agricultural Modernization, 2016,37 (3): 416-422 .

Reference to a book: 
[2] Kong Fantao, Li Huishang, Wang Shengwei, Qin Bo. 2015 Vegetable Market Situation Analysis and Outlook in China [J] China Vegetables, 2016,323 (1): 8-12.

[3] Kong Fantao, IOT Rranch --- New Transformation of Modern Livestock Husbandry [J] Market Weekly, 2014, (48): 36-39.

[4] Zhang Xinmin, Situation and Outlook of China Agricultural Informatization Development [J] Agricultural Economy, 2011, 08: 35-37. 